\title{
Les politiques nationales de développement urbain durable en France à l'épreuve des expérimentations locales : le cas des écoquartiers
}

\author{
> Michael Fenker* et Jodelle Zetlaoui-Léger \\ LET UMR LAVUE (CNRS 7218), École Nationale Supérieure d'Architecture \\ Paris-La-Villette, 144 avenue de Flandre, 75019 Paris
}

\section{Résumé}

Cette contribution propose un éclairage sur "la fabrique des politiques publiques» dans le domaine de l'action urbaine sous deux angles distincts, mais indissociables. L'un concerne les modalités d'élaboration et de diffusion d'une politique nationale en faveur de la "ville durable», en précisant la façon dont l'État a mis en place, à travers l'incitation à la réalisation " d'écoquartiers », un nouveau « dispositif technique et social » pour encourager et évaluer une transformation des pratiques dans l'aménagement urbain en France. L'autre porte sur la réalisation de ces opérations par les collectivités locales, en s'intéressant à la façon dont celles-ci conçoivent et réalisent leurs projets, dans un processus d'ajustement entre les ambitions de la politique nationale et les enjeux politiques et sociaux propres à leur territoire.

II apparaît ainsi que la démarche de labellisation «ÉcoQuartier » qui cristallise un axe majeur de la mise en œuvre de la politique nationale «ville durable», s'est trouvée au cœur de dynamiques d'articulation entre des mouvements descendants et ascendants, de coopérations transversales entre organismes ministériels et collectivités, de concertations impliquant une diversité d'acteurs, y compris ceux de la société civile. L'analyse d'un corpus de 187 opérations d'écoquartiers montre que les collectivités locales disposent de capacités inégales pour définir et concilier les différents objectifs à poursuivre, et plus largement, pour mettre en place de nouvelles modalités de gouvernance et de pilotage permettant d'impliquer les citoyens dans la conception des projets, de composer avec les politiques nationales et de peser sur celles-ci. La contribution montre également comment la démarche partenariale originale mise en place à l'occasion du programme "ÉcoQuartier » met à l'épreuve un fonctionnement des administrations centrales encore très hiérarchisé et sectorisé en France.

(c) 2017 IDMP/Lavoisier SAS. Tous droits réservés

Mots clés : écoquartiers, développement urbain durable, pilotage et gouvernance des projets urbains, participation citoyenne, politiques de développement durable, Grenelle de l'Environnement.

\footnotetext{
*Auteur correspondant : michael.fenker@paris-lavillette.archi.fr doi :10.3166/pmp.34. 2017.0005 @ 2017 IDMP/Lavoisier SAS. Tous droits réservés
} 


\section{Abstract}

National sustainable urban development policy in France challenged by local experiments - the case of econeighborhoods. This contribution proposes throwing light on the « construction of public policies » within the framework of urban action. This is approached from two separate yet indissociable points of view. One concerns the ways of developing and disseminating a national policy in favour of the "sustainable city». This approach specifies the way in which the State, through encouragements to construct « econeighborhoods », has introduced a new «technical and social device» to encourage and evaluate a transformation of practices in French urban development. The other concerns the implementation of these operations by local authorities. It involves examining the way in which these authorities design and construct their projects within a process of adjustment between the ambitions of national policy and the political and social issues specific to local territories.

It seems therefore that the «EcoNeighborhood » labelling approach crystallising a major aspect in the introduction of the national « sustainable city » policy finds itself lying in the heart of the articulation dynamics between ascending and descending movements, transversal cooperation between ministerial bodies and local authorities, and collaboration involving a diversity of players, including those from civil society. An analysis of a corpus of 187 econeighborhood operations reveals that local authorities have differing capacities in the defining and reconciling of the various objectives to be pursued. More generally, this concerns introducing new methods of governing and management that allow citizens to become involved in the design of projects, to work with national policies and to have an influence on the latter. The contribution also reveals how the innovative partnership approach set up for the « ecoNeighborhood » programme puts to the test the operation of central administrations that remain particularly hierarchical and sectorial in France.

( 2017 IDMP/Lavoisier SAS. Tous droits réservés

Keywords: Econeighborhoods, sustainable urban development, management and governing of urban projects, citizen participation, sustainable development policies, Grenelle environment forum.

\section{Introduction}

Depuis le milieu des années 2000,1'aménagement urbain est devenu un objet privilégié dans différentes sphères, politiques, militantes, scientifiques, et opérationnelles en France pour penser et organiser l'ancrage territorial du développement durable. Dans cette perspective, les « quartiers durables » ou «écoquartiers », dont la réalisation est promue par l'État depuis le Grenelle Environnement (GE), sont considérés comme un cadre propice pour expérimenter et diffuser les principes et les valeurs de « la ville durable ». Ils sont de fait porteurs de nouvelles attentions dans la manière de fabriquer de l'urbain, qui visent notamment à de meilleures articulations entre échelle globale et échelle locale dans les politiques publiques d'aménagement, au développement d'approches intersectorielles, à une implication des citoyens aux processus de décision publique concernant leur cadre de vie.

Si on a vu émerger dès le début des années 90 dans plusieurs pays d'Europe du Nord, des expériences de « quartiers durables », à l'initiative de collectivités ou de groupes d'habitants mobilisés, il a fallu attendre en France la fin de la première décennie des années 2000, et plus précisément, les orientations législatives liées au Grenelle de l'Environnement, pour 
que ce type d'opérations soit conçu et valorisé comme tel. En effet, les changements de paradigmes dans le domaine de l'aménagement de l'espace et de la production du cadre bâti ont toujours eu besoin en France d'une impulsion forte de la part des autorités centrales ou supra-nationales, pour se déployer ensuite localement. Cependant, la démarche de labellisation «ÉcoQuartier » se caractérise par de nouvelles préoccupations exprimées par le Ministère de l'Écologie, du Développement durable, des Transports et du Logement, initiateur de cette politique, pour mettre en place un dispositif plus horizontal de gouvernance et de diffusion des résultats d'expérimentations. L'analyse de ces nouvelles formes de politiques publiques et de collaboration entre représentants des administrations centrales et acteurs locaux constitue le propos de cet article.

Afin de stimuler les initiatives, de repérer des « bonnes pratiques » et de les valoriser, le Ministère de l'Écologie a mis en œuvre deux appels à projets « ÉcoQuartier » ${ }^{2}$ en 2009 et 2011, préfigurant un processus de labellisation engagé en 2012. Dans ce cadre, plus de 400 collectivités ont pu faire connaître leurs expériences et échanger sur celles-ci au sein d'un «Club ÉcoQuartier », animé par le Ministère avec le concours de partenaires institutionnels et de chercheurs. Comment apprécier les résultats de ces incitations, au niveau des politiques et pratiques d'aménagement des collectivités locales, au regard des objectifs visés par les politiques nationales? Les travaux qui contribuent depuis 2014 à l'élaboration d'une « Méthode nationale d'Évaluation » des projets d'ÉcoQuartiers en France menés au sein des différentes instances de ce club, et en particulier de son Comité scientifique, en fournissent une indication. Ils mettent en exergue l'importance de s'intéresser, au-delà des transformations du cadre bâti et aménagé, aux démarches et processus développés à l'occasion de ces opérations afin d'évaluer les effets et la portée de l'action publique (Souami, 2011; Zetlaoui-Léger, 2013). Il s'agit de comprendre comment et à quel degré les stratégies de projet urbain sont intégratrices des intentions politiques nationales et locales. Nous avancerons l'hypothèse que la démarche ÉcoQuartier, considérée ici comme un « instrument » de politique publique (Lascoumes et Le Gales, 2004; Halpern et al., 2014), a été l'occasion de voir s'exprimer de nouveaux rapports entre l'État et les collectivités, entre « gouvernants » et «gouvernés », dans le champ de l'urbanisme, mais que la pérennisation de ceux-ci se heurterait à plusieurs types d'obstacles que nous chercherons à identifier.

Dès la mise en place du GE, des voix s'étaient élevées pour s'interroger sur la solidité du processus de concertation politique qui conduisait à la formalisation d'engagements en faveur d'un développement durable. Elles se questionnaient également sur les effets des décisions gouvernementales dans la réalisation des ambitions affichées. Une des incertitudes exprimées concernait la tenue dans le temps et la « réplicabilité » du processus de concertation mené au sein du GE, qualifié de dynamique et de relativement productif, ainsi que sa capacité à s'articuler aux instances de la démocratie représentative et au fonctionnement institutionnel classique en France (Lascoumes, 2011). D'autres analyses s'intéressaient à la diffusion et l'appropriation des injonctions et cadres normatifs énoncés au niveau national, considérant le territoire local, avec ses enjeux politiques propres, comme un « lieu d'ajustement au regard d'une norme » et identifiant la force d'inertie des professionnels comme un frein à la diffusion de nouvelles pratiques nécessaires à la poursuite des objectifs (Villalba, ed., 2009).

\footnotetext{
${ }^{2}$ On distinguera dans le texte la notion générique d'écoquartier de celle associée au label créé par le ministère avec l'orthographe ÉcoQuartier.
} 
Dans le prolongement de ces interrogations, une analyse de « la fabrique des politiques publiques » dans le domaine de l'action urbaine devrait alors être menée sous deux angles distincts, mais indissociables :

- l'un concerne les modalités d'élaboration et de diffusion d'une politique nationale en faveur de la « ville durable », en précisant la façon dont l'État a mis en place un nouveau « dispositif technique et social » pour encourager, accompagner et observer une transformation des pratiques dans l'aménagement urbain en France, de l'élaboration d'un référentiel à la mise en place d'une Méthode nationale d'Évaluation pour mesurer les progrès accomplis;

- l'autre porte sur la réalisation d'écoquartiers par les collectivités locales, en s'intéressant à la façon dont celles-ci définissent leurs ambitions en matière d'urbanisme durable, organisent le montage et la conduite de leur projet en lien plus ou moins étroit avec les directives et les représentants des instances nationales. À cette échelle de l'analyse, le développement durable, en ce qu'il requiert une vision globale, holistique des problèmes en jeu à un niveau local (Berke, 2002), permet de poser avec acuité la question de l'interdépendance et du dialogue entre citoyens, « experts patentés » et élus locaux. Une seconde hypothèse mise en jeu dans cette contribution, consiste à avancer que l'engagement au plan national et local, dans des démarches et objectifs ambitieux en matière de développement durable, fidèle aux principes du cadre de référence national « Agenda 21 » repris par la loi dite Grenelle $\mathrm{II}^{3}$, dépendrait non seulement des impulsions initiales qui leur sont données par leurs acteurs respectifs, mais aussi de la gestion dans la durée de leurs interactions à l'un et l'autre niveau. Celle-ci se jouerait notamment dans la tension entre d'une part, une performativité attendue par l'État qui peut glisser vers la normalisation des processus et des résultats, et d'autre part, une nécessité pour les collectivités de s'appuyer sur les spécificités d'un contexte local, de poursuivre des expérimentations déjà initiées sur leur territoire ou qu'elles souhaiteraient conduire à l'occasion d'une politique publique nationale.

Cette contribution est structurée en trois parties. Une première précise comment l'État a défini et mis en place un système de déclinaisons et de suivi de sa politique en faveur d'un développement urbain durable, dans un contexte d'amplification du processus de décentralisation. Une deuxième partie examine les démarches développées par les collectivités locales pour se saisir des politiques nationales dans le cadre de leurs projets urbains, en y associant à des degrés divers la société civile (associations, habitants, usagers). Ces deux premiers volets s'appuient sur deux recherches que nous avons menées sur la fabrication des premiers écoquartiers en France (Zetlaoui-Léger, dir., 2013 ; 2014) donnant lieu à des enquêtes statistiques et des analyses qualitatives de 187 opérations. Une troisième partie porte sur le processus d'élaboration de la « Méthode nationale d'Évaluation » des écoquartiers dans lequel les auteurs de cette contribution (Fenker et Zetlaoui-Léger, dir., 2015) se sont trouvés en situation d'observation participante, au cœur même de la mise en œuvre

\footnotetext{
${ }^{3}$ Celui-ci énonce cinq principes majeurs pour "I'action publique durable": "transversalité", "organisation du pilotage", "participation des citoyens", stratégie d'amélioration continue", "évaluation partagée". Cf. "Cadre de référence pour les projets territoriaux de développement durable et agendas 21", Circulaire du 13 juillet 2006 du MEDD, et Loi du 12 juillet 2010 dite Grenelle 2 portant engagement pour l'environnement (Titre VI "Gouvernance", Chapitre V, "Projets territoriaux de développement durable", articles 252-254.
} 
d'une politique publique ${ }^{4}$. L'ensemble de ce processus sera considéré comme un moment particulier d'une volonté portée par quelques représentants d'administrations centrales d'articuler les politiques nationales et les pratiques locales. Il met à l'épreuve les instances ministérielles dans leur capacité à prendre en compte les retours d'expériences des collectivités, à élargir leur mode d'appréciation des résultats à des indicateurs plus qualitatifs et éloignés des postures positivistes, à construire ceux-ci avec les représentants de la société civile et des acteurs privés de l'aménagement.

Nous chercherons ainsi à appréhender les conditions qui ont rendu possible lors de la mise en place de la démarche nationale ÉcoQuartier, l'instauration d'une telle dynamique, mais également les difficultés rencontrées cinq ans après son lancement pour prolonger ces principes.

\section{La mise en place de la démarche ÉcoQuartier}

Les projets d'urbanisme constituent une forme particulière d'action publique (Pinson, 2004). Bien qu'ils soient le produit de négociations entre acteurs publics et privés, ils résultent en France d'une volonté politique et plus précisément d'une maîtrise d'ouvrage urbaine publique supposée être porteuse d'un « intérêt général » (Vanier, 2011). Les intentions en matière d'aménagement sont également censées s'intégrer dans des stratégies de territoires définies à plusieurs échelles par des collectivités dans des documents d'urbanisme (Bourdin, 2005). Depuis la promulgation de la Loi Solidarité et Renouvellement Urbain en 2001, l'État central n'a plus vocation à imposer aux collectivités de directives précises en matière d'aménagement urbain, néanmoins, il reste toujours présent à travers ses organismes déconcentrés pour veiller à « la cohérence » entre les différents documents d'urbanisme élaborés et au fait que ceux-ci tiennent bien compte des objectifs de politiques nationales énoncés dans de grandes lois d'orientations.

En souscrivant à l'Agenda 21 à l'issue du sommet de Rio de 1992, puis en ratifiant la Convention européenne d'Aarhus en 2002, la France a officiellement adhéré au principe d'un développement durable qui croise enjeux environnementaux, économiques et sociaux. Cependant, les collectivités locales ne se sont véritablement engagées dans cette démarche qu'après la mise en place d'un cadre juridique incitatif (Emelianoff, 2007). Une grande partie des premiers projets d'écoquartiers français correspondent à des opérations qui n'avaient pas forcément été envisagées dès le départ avec des ambitions fortes en matière de développement durable. Celles-ci ont souvent été introduites au fil du déroulement de ces projets, dans de très rares cas sous l'impulsion de groupes d'habitants mobilisés sur les questions environnementales, mais surtout à partir de 2008, lorsque l'État met en place une politique nationale en faveur de la Ville durable.

Ainsi, la démultiplication de projets d'écoquartiers est consécutive à la formulation d'injonctions réglementaires, à la suite des débats organisés à l'occasion du Grenelle de l'Environnement en 2007. La loi de Programmation dite Grenelle 2 stipule : «L'État encouragera la réalisation, par les collectivités territoriales, d'opérations exemplaires d'aménagement durable des territoires. Il mettra en ouvre un plan d'action pour inciter les collectivités

\footnotetext{
${ }^{4}$ Les auteurs étaient membres du Comité Scientifique de la démarche EcoQuartier dès sa création en 2009. Ils ont mené dans ce cadre plusieurs études d'évaluation de la démarche EcoQuartier en cours.
} 
territoriales, notamment celles qui disposent d'un programme significatif de développement de l'habitat, à réaliser des écoquartiers avant 2012 en fournissant à ces collectivités des référentiels et une assistance technique pour la conception et la réalisation des projets... $\gg^{5}$

De manière plus générale, le GE apparaît comme fondateur d'une nouvelle approche en France dans la façon de définir et de mettre en œuvre des politiques publiques qui vont largement inspirer l'organisation de la démarche nationale ÉcoQuartier. Il incite à la recherche d'intérêts partagés et non catégoriels, ce qui se traduit, entre autres, par la fusion entre les administrations chargées de l'Écologie et de l'Équipement au sein d'un même ministère qui dirigera la démarche nationale ÉcoQuartier. Il plébiscite des modes de concertation intégrant une diversité d'acteurs, dont ceux de la société civile. Les collectivités locales sont appelées à jouer un rôle central dans ces démarches, l'État se donnant un statut plutôt d'accompagnateur et se défendant de vouloir reprendre le contrôle de la planification urbaine.

Cette ambition politique conduit à la création d'un Bureau de l'Aménagement Opérationnel Durable (AD4) au sein du Ministère de l'Écologie et du Développement Durable qui se voit confier la mise en œuvre de la démarche ÉcoQuartier. Cette entité est placée sous la responsabilité de la Direction Générale de l'Aménagement du Logement et de la Nature (DGALN), et plus directement, de la Direction de l'Habitat, de l'Urbanisme et des Paysages (DHUP). Son responsable, qui malgré son jeune âge et son statut d'architecte, ingénieur des Travaux Publics de l'État, bénéficie de la confiance que lui accorde la sous-direction de l'Aménagement Durable et d'un mouvement de recomposition administrative (départs à la retraite, fusions entre directions), obtient la constitution d'une petite équipe pluridisciplinaire. La démarche va ainsi pouvoir se construire au croisement d'un ensemble de politiques sectorielles directement concernées par le développement urbain durable. Pour autant, peu de moyens sont consacrés à cette action. Les membres de la structure sont néanmoins particulièrement motivés par leur mission : mettre en place un instrument de pilotage national d'une politique publique pour faire la promotion de la Ville durable.

Pour inciter les collectivités à s'engager dans des actions en faveur de la Ville durable, plusieurs appels à projet sont prévus, autour de différentes thématiques et échelles d'aménagement : «ÉcoQuartier»; «ÉcoCités », « Transports collectifs », « Nature en ville ». La pratique du concours et de l'appel à projets est inscrite dans la culture politique et technique française. Elle est particulièrement bien connue des acteurs du champ de l'aménagement où elle correspond aux modes de dévolution des marchés de maîtrise d'œuvre des opérations de constructions publiques que l'État a cherché à systématiser à partir des années 70 (Epron, 1992). «L'émulation » est également un mode de gouvernance couramment utilisé par les administrations françaises dans la conduite des politiques publiques (Epstein, 2013 ; Baudot, 2014), pour sélectionner sur projet ceux à qui seront distribuées des subventions. Or, l'appel à projets ÉcoQuartier ne prévoit aucun soutien financier aux collectivités primées. L'équipe de l'AD4 ne se démobilise pas pour autant et cherche d'autres motifs incitatifs.

L'AD4 doit d'emblée composer avec des injonctions qui peuvent paraître paradoxales. D'un côté, l'État central veut encourager des « bonnes pratiques », comprendre ainsi comme cohérentes avec les principes que l'État préconise dans ses lois d'orientations, celles du Grenelle notamment. La mise en place d'un « référentiel ÉcoQuartier » en 2012, prévue

\footnotetext{
${ }^{5}$ Article 7-III-Dispositions relatives aux objectifs, LOI n ${ }^{\circ} 2009-967$ du 3 août 2009 de programmation relative à la mise en cuvre du Grenelle de l'environnement.
} 
dans la loi de 2009, s'inscrit dans la même logique. On retrouve dans cette déclaration la façon dont l'État entend assurer son rôle régalien traditionnel de garant de « l'intérêt général » (Rosanvallon, 2008), en définissant un cadre incitatif censé déterminer un niveau d'ambition à atteindre par les collectivités. D'un autre côté, la décentralisation et le concept même de développement durable invitent à une contextualisation des démarches. L'AD4 prend d'ailleurs d'emblée plusieurs précautions à cet égard.

Dès le départ, la notice explicative du premier appel à projets ne fournit pas une définition canonique trop précise de ce qu'elle considère comme pouvant être un ÉcoQuartier et propose finalement aux collectivités qui seront candidates de construire la leur en fonction des objectifs qu'elles poursuivent dans leurs opérations. Il s'agit d'une différence importante par rapport à d'autres procédures de labellisation. Les promoteurs de la démarche souhaitent que cette notion se construise progressivement, dans un jeu d'allers-retours avec les communes impliquées. Ils veulent aussi polariser l'attention sur les phases amont d'élaboration des projets. Cette position est clairement assumée : "le ministère souhaite lancer, avec des collectivités, une démarche Écoquartier à la fois pour en consolider le contenu, mais aussi pour diffuser largement le processus de projet et les procédures de montage, de financement et de suivi des objectifs ».

Plusieurs objectifs sont ainsi poursuivis : consolider ce qui peut être entendu par aménagement urbain durable, et plus précisément par la notion d'écoquartier en se basant sur des expériences concrètes; inciter les collectivités à expérimenter et à en tirer des enseignements pour leur politique urbaine locale; les inviter à partager leurs expériences avec d'autres à l'échelle nationale. L'AD4 propose à cet effet la constitution d'un Club ÉcoQuartier qui leur permettra de bénéficier d'un ensemble d'actions pour progresser dans leurs réflexions. L'effet « vitrine » au plan national et international est également l'un des enjeux de la démarche. Il est poursuivi à travers la mise en place d'un Palmarès décerné à l'issue de chaque appel à projets afin de donner une certaine ampleur médiatique au dispositif. La première édition du concours « ÉcoQuartiers » 2009 va ainsi cristalliser l'attention des médias.

Cet appel à projets s'organise à partir de la constitution d'une notice qui explique les objectifs et règles du concours et donne des indications sur la façon dont les dossiers seront évalués. Il a été préparé par l'AD4, en accord avec sa direction et le cabinet du ministre. Dans une première partie, les collectivités sont censées présenter la nature de leur opération et l'équipe en charge du projet; la deuxième est consacrée aux « intentions que l'équipe projet s'est données sur chacun des trois piliers du développement durable »; la troisième, doit évoquer «le (les) thème(s) d'excellence dans lequel l'équipe souhaite s'investir parmi les 7 proposés ».

La dimension « pédagogique » de la consultation apparaît clairement dans ce document adressé aux collectivités. Il ne s'agit pas seulement de valoriser des expériences mais aussi de montrer aux collectivités ce qui est attendu d'une démarche d'aménagement urbain durable. Pour cela, la notice explicative propose pour chaque partie de dossier à remplir, une grille où sont présentés par «pilier» du développement durable, des thèmes, des enjeux, des objectifs pour lesquels, les collectivités doivent expliquer les actions qu'elles ont mises en place. La formulation des sujets doit aussi bien les guider à remplir le dossier, que les mettre sur la piste de ce que l'État attend d'eux. Les questions sont souvent très ouvertes, lorsqu'elles demandent aux collectivités comment elles ont essayé d'atteindre un objectif, mais elles sont parfois aussi plus que directives quand la réponse attendue est contenue dans l'interrogation. La démarche paraît de ce fait assez inductive. 
Par ailleurs, transparait dans la présentation du dossier, une vision assez « orientée » du type de projet que le ministère chercherait à valoriser. Cette impression va se confirmer à l'annonce des résultats du Palmarès au printemps 2009. Les dimensions environnementales et éco-techniques liées aux formes urbaines, à l'éco-construction et à la gestion de l'eau, des déchets, de la biodiversité, de la mobilité, des énergies, sont appréhendées comme les plus importantes dans la fabrication d'un écoquartiers. La valorisation de ces «thèmes d'excellence » se fait inévitablement au détriment non seulement des piliers sociaux et économiques constitutifs du développement durable, mais plus largement d'une vision intégrée des dimensions qui le structurent. On peut également remarquer la faible attention apportée à la « gouvernance » des projets, et en particulier au rôle joué par la société civile, aspect pourtant considéré comme fondamental dans tous les traités internationaux sur le développement durable que la France a ratifiés. Ces orientations sont le résultat d'inflexions demandées par le cabinet du Secrétariat au Logement qui supervise la consultation.

L'AD4 ne s'attend pas à obtenir un grand nombre de réponses, compte tenu de l'importance du dossier à réaliser et de l'absence de subventions à proposer. Il va finalement recevoir 160 dossiers, à la grande surprise également de sa sous-direction qui lui propose alors de se faire assister pour l'évaluation par un cabinet de conseil. L'équipe de l'AD4 refuse, préférant mettre en place un dispositif d'évaluation plus transparent et pluriel à trois niveaux : une expertise interne au ministère, une expertise externe, un avis de représentants de la DDE concernée. Sont ainsi impliqués des techniciens des administrations centrales, déconcentrées et des personnalités indépendantes (membres d'associations d'élus et d'organisations professionnelles, universitaires, experts libéraux). L'équipe défend plusieurs intentions : assurer un portage collectif de la démarche en y acculturant une diversité d'acteurs, se placer dans une dynamique de capitalisation des enseignements de la consultation dans la durée, rendre la qualité de l'évaluation peu contestable. Ce dispositif d'une grande ampleur est finalement accepté par la sous-direction de l'Aménagement Durable. Le Bureau AD4, renforcé par quelques stagiaires, en fait un véritable défi, comprenant qu'il s'agit de mettre en place un dispositif assez inédit dans l'administration centrale. L'engouement au sein du Bureau est tel entre les différents collaborateurs très impliqués sur l'objet même de la consultation que les difficultés parviennent à être surmontées.

Ce premier appel à projets est considéré comme un succès au ministère et par les médias spécialisés auprès des professionnels de l'aménagement. Outre le niveau de participation, signe pour l'AD4 de l'intérêt que suscite l'aménagement durable auprès des collectivités et, pour le ministère, du bien-fondé de la politique publique qu'il a engagée, la répartition équilibrée des dossiers entre des villes de tailles très différentes (de moins de 500 habitants à plusieurs milliers d'habitants) accrédite la pertinence de la consultation. La triple expertise a manifestement donné un gage de sérieux à l'ensemble de la procédure. Si les pressions politiques n'ont pas manqué pour soutenir tel ou tel projet, la qualité de la préparation du jury a rendu difficiles des choix seulement partisans. Ainsi, les résultats n'ont pas été fondamentalement contestés.

Néanmoins, les observateurs avertis, en particulier les chercheurs et experts qui travaillent depuis plusieurs années sur le développement urbain durable à l'échelle internationale, se montrent assez dubitatifs au regard des expériences que met en lumière le concours ÉcoQuartier, y compris de celles qui sont primées : "contrairement aux premiers écoquartiers européens construits dans des circonstances, avec des délais et des moyens exceptionnels, 
les projets d'écoquartiers français ne relèvent ni d'une réglementation "expérimentale » ni d'un cadre opérationnel spécifique » (Lefèvre, Sabard, 2009). Beaucoup reprochent aux collectivités de revendiquer abusivement l'appellation « d'écoquartiers » ou de « quartiers durables » pour des projets qui s'apparenteraient principalement à une juxtaposition de bâtiments vitrines à basse consommation énergétique, et de "s'exonérer de réfléchir à la démarche de conception urbaine $»^{6}$ prenant la mesure de toute la transversalité de la notion de développement durable, et des articulations entre les différentes échelles de l'aménagement. Les chercheurs sont également critiques sur l'organisation du concours; ils n'ont pas manqué de relever dans le dossier de consultation, la mise en retrait des dimensions sociales et économiques, ainsi que le peu d'importance accordé au pilotage des projets et aux problématiques de participation citoyenne (qui ont été intégrées au «pilier social et sociétal »), au profit d'une focalisation sur les éco-techniques environnementales.

Loin de déstabiliser les promoteurs de la démarche au sein du Bureau AD4, ces critiques les confortent pour faire évoluer la démarche ÉcoQuartier dans le sens d'une meilleure prise en compte de ces aspects, par rapport auxquels ils n'avaient en fait pas réussi auparavant à convaincre leur hiérarchie plus encline à valoriser les aspects technico-environnementaux. Ils comptent bien tirer parti de la légitimé que leur a conféré le succès du premier appel à projets pour mettre en place une réflexion plus ambitieuse, s'appuyant sur les retours des collectivités et sur des recherches. L'objectif est d'étendre les échanges et l'apprentissage entre acteurs sur la fabrication de la Ville durable. Ils obtiennent à cet effet début 2010 la création d'un Comité Scientifique, la mise en place d'un réseau d'informations en ligne et celle d'un dispositif d'assistance ponctuelle gratuite aux collectivités souhaitant s'engager dans une démarche ÉcoQuartier. Des groupes de réflexions nationaux ouverts aux collectivités, aux professionnels de l'urbain sont également créés autour de quatre grandes thématiques transversales.

Le Comité scientifique créé le 4 mai 2010 et auquel notre laboratoire est associé, est un organe consultatif composé d'universitaires et de représentants d'organismes institutionnels publics et parapublics (ADEME, CERTU futur CEREMA, CSTB, PUCA). Il a pour mission de contribuer à la réflexion sur les problématiques majeures associées à l'aménagement urbain durable, à la construction du Référentiel et d'une Méthode nationale d'Évaluation, ainsi qu'à la réalisation d'un Observatoire des ÉcoQuartiers. Ses membres ont été choisis en raison de leur implication dans des recherches ou études sur le développement durable en milieu urbain ou rural. Partagés entre la volonté de valoriser leur travaux dans une politique publique en train de se fabriquer et la crainte d'être instrumentalisés, les chercheurs du Comité scientifique annoncent dès la première séance, qu'ils ne souhaitent pas élaborer la grille ni le référentiel. Ils sont toutefois prêts à alimenter les réflexions à ce propos, sur un mode critique mais constructif, se basant sur leurs résultats de recherche. Ils redoutent d'être associés à la construction d'une « doctrine » s'imposant d'en haut, à travers un référentiel dont ils redoutent l'effet normatif. La direction de l'AD4 accepte cette position et est d'autant plus à l'aise avec la situation, qu'elle envisage de faire évoluer la grille et de construire le Référentiel à partir des résultats de travaux de recherche et de différentes concertations.

\footnotetext{
${ }^{6}$ CHARLOT-VALDIEU (Catherine), "Les villes abusent de l'appellation d'éco-quartier", Le Monde, Planète, Mis en ligne le 20/03/2009, http://www.lemonde.fr/planete/article/2009/03/20/les-villes-abusent-de-Iappellation-d-eco-quartier_1170515_3244.html
} 
Pour mener à bien ses missions, l'AD4 doit accepter la demande de sa direction de se faire assister par deux grands cabinets de conseil. Inquiète d'une possible externalisation de la réflexion, l'équipe insiste sur le fait que le ministère continuera à piloter la démarche sans s'effacer derrière les consultants. Elle pose également les grands principes d'organisation de la réflexion à mener sur la forme que devra prendre le référentiel : « mise en œuvre d'une concertation avec différents types d'experts du sujet dans le cadre de séminaires, campagne de tests avec les acteurs locaux, arbitrages, justification des choix auprès des participants à la démarche $»^{7}$. Le cabinet du secrétariat d'État au Logement s'attendait à la production d'une nouvelle norme, sur le modèle de celle produite par l'AFNOR (ISO 37101) sur le « Management des Quartiers Durables et Résilients ». Or l'équipe de l'AD4 préfère une approche qui place les acteurs dans des dynamiques d'apprentissage tout au long d'un projet. Ne cherchant pas à imposer un point de vue et à s'opposer à sa direction, elle préfère laisser la question à débattre. Consulté à ce sujet fin 2010, le Comité scientifique souligne que «le processus de reconnaissance devant porter avant tout sur une démarche innovante, expérimentale, et non un produit ${ }^{8}$ L'idée d'attribuer un "label, permettant de garder une certaine souplesse dans la définition, tout en assurant une bonne visibilité des ÉcoQuartiers réalisés $»^{9}$ est partagée et se diffuse.

Un Comité de Préfiguration du label est créé, regroupant élus, représentants des collectivités, d'entreprises privées, d'organisations professionnelles, d'ONG, d'associations, d'universitaires. La réflexion aboutit en 9 mois, après une démarche intense et soutenue, par une étroite collaboration entre l'équipe de l'AD4 et ses consultants, à l'idée d'un « processus de labellisation » en 3 étapes. Le Comité de Préfiguration du label a conclu des différents débats organisés, d'une part, qu'il ne fallait pas chercher à normer la ville, d'autre part, qu'il n'était pas pertinent de décerner un label à un projet de manière définitive à partir d'une évaluation ponctuelle menée par des experts sur des bases principalement déclaratives. Il s'agira plutôt de mettre en place un processus d'évaluation à plusieurs moments de la conception et de l'aménagement d'un quartier. L'attention devra porter « aussi bien sur la démarche que sur le contenu des réalisations ». Les conclusions du Comité de Préfiguration révèlent à travers cette approche trois grands types de préoccupations : "permettre la contextualisation des objectifs nationaux aux territoires et aux types de projets; capitaliser les connaissances, former et sensibiliser les parties prenantes; s'appuyer sur les acteurs locaux ». La perspective de faire également porter la labellisation sur la « vie du quartier », idée défendue par le Comité scientifique, a été retenue.

Ce processus réflexif, inter-institutionnel et concerté se prolonge dans la réactualisation de l'appel à projets 2011, dont la grille évolue significativement, à partir d'une évaluation critique du premier appel à projets que réalisent les différentes composantes du Club ÉcoQuartier : les groupes de réflexions thématiques associant des collectivités, des professionnels de l'aménagement et des grands groupes industriels du BTP, le comité scientifique et les relais techniques locaux du ministère. Le concours 2011 reçoit 393 candidatures, l'année suivante, 80 collectivités sont considérées comme « engagées dans la labellisation » (Étape 2 du processus).

\footnotetext{
${ }^{7}$ Interview avec le directeur de l'AD4 de l'époque, 19/11/2015.

${ }^{8}$ Compte rendu Comité scientifique 23 novembre 2010.

${ }^{9}$ Compte-rendu du Comité scientifique du 22 février 2011.
} 
Il ressort de cette réflexion sur la « forme du Référentiel » qui a abouti à un principe de labellisation par étape, un assez fort consensus entre ses différents contributeurs, malgré le scepticisme de certains pour des motifs très différents au départ. La méthode de travail qui a été adoptée, concertée, itérative et transparente, faisant dialoguer acteurs nationaux et locaux, chercheurs et praticiens, représentants d'organismes institutionnels et de la société civile, y a manifestement beaucoup contribué. Elle apparaît comme une innovation dans la construction d'une politique urbaine portant sur des processus et procédures d'aménagement qui s'élaborent plus traditionnellement dans le cadre de concertations fermées par le nombre et le profil des participants. Le fait que la démarche soit ouverte aux collectivités représentées par des acteurs très divers, et s'appuie sur des retours d'expériences, portées directement par des élus et des praticiens, ou sur la base de recherches et d'études, est également peu conventionnel dans les pratiques institutionnelles en France. Le caractère extrêmement transversal de la collaboration entre des administrations centrales relevant de différentes directions, puis de plusieurs ministères ${ }^{10}$, que parvient à préserver l'AD4 jusqu'en 2014, est plus encore, exceptionnel.

\section{Étapes de la mise en œuvre d'une politique nationale ÉcoQuartier}

Octobre 2008 : Mise en place du Plan ville durable présenté par Jean-Louis Borloo, Ministre de l'écologie, de l'énergie, du développement durable et de la mer (MEEDDM), dans le cadre des réflexions du Grenelle de l'Environnement.

3 Août 2009 : Promulgation de la loi Grenelle 1. Toutes les communes qui ont des programmes de développement de l'habitat significatif doivent avoir au moins un écoquartier avant 2012.

2009 : Premier appel à projets ÉcoQuartiers du Ministère de l'Écologie, du Développement Durable et de l'Énergie

2010 : Création du Comité scientifique et du Club ÉcoQuartiers

2011 : Second appel à projets ÉcoQuartiers du Ministère de l'Écologie, du Développement Durable et de l'énergie

2012 : Début du processus de labellisation ÉcoQuartiers - Test et attribution des premiers labels

2013 : Début de l'élaboration d'une Méthode nationale d'Évaluation des ÉcoQuartier 2014 : Mise en place d'une mission de préfiguration d'un Institut pour la Ville Durable (IVD)

\section{Le déclinaison des politiques publiques nationales au niveau des collectivités locales}

La perspective d'une forme de reconnaissance nationale des opérations des collectivités locales à l'occasion des deux appels à projets du Ministère de l'Écologie et du Développement Durable s'est révélée particulièrement incitatrice. Pour répondre à ces consultations, les maîtres d'ouvrages publics se sont mis, soit à requalifier d'anciens projets sous ce vocable,

${ }^{10}$ En 2010, les domaines de l'Énergie, de l'Espace Rural et de l'Aménagement du Territoire, ne sont plus intégrés au Ministère de l'Écologie et du Développement Durable, des Transports et du Logement. En 2012, celui du Logement est de nouveau rattaché à un autre Ministère en charge de l'Égalité des Territoires. 
soit à se lancer dans de nouvelles opérations sous cet intitulé. La crise immobilière de 2008 a également pu encourager certaines municipalités à y répondre, percevant sans doute l'obtention d'un prix par le ministère comme un moyen de valoriser et de rendre attractive leur opération, dans un contexte où nombre de promoteurs immobiliers se désengagent.

Un autre aspect de la réception des politiques nationales par les collectivités locales est celui de la stabilisation du vocabulaire des élus, techniciens et universitaires pour désigner ces projets (Grudet, 2015). L'appellation ÉcoQuartier semble s'imposer avec la diffusion d'une pensée où les préoccupations environnementales ne sont plus dissociées des orientations économiques et sociales. Néanmoins, on constate que certaines collectivités ont réagi avec circonspection à l'engouement national pour cette notion, y voyant une logique de marketing urbain qui risquerait de réduire le développement urbain durable à la promotion d'un nouvel objet immobilier, principalement axé sur la valorisation de performances écotechniques, et de faire l'impasse sur l'ambition d'un changement des modes de vie.

Mais au-delà des questions d'appellation, comment les collectivités se sont-elles saisies de la démarche ÉcoQuartier, et plus largement des modes de gouvernance préconisés à travers le concept de développement durable, dans l'élaboration de leurs projets ? Les résultats que nous avons obtenus à partir de la mise en œuvre d'un dispositif d'investigation basé sur plusieurs enquêtes menées aux plans national et local auprès d'une diversité d'acteurs (Zetlaoui-Léger dir., 2013 ; 2014), tendraient à montrer que les opérations du concours ÉcoQuartier ont bien été l'occasion pour un nombre significatif de maîtres d'ouvrage (troisquarts des opérations analysées) d'aborder différemment la place des habitants, organisés ou non en associations, dans les processus de projet. Si l'injonction à la concertation citoyenne était explicitée dans certains textes réglementaires depuis les années 90, nos recherches montrent qu'une grande partie des maîtres d'ouvrage s'en était toujours tenue jusque-là à une concertation dite « réglementaire », se limitant à une ou deux réunions publiques. Cela conforterait l'idée que la conjonction entre des enjeux environnementaux, considérés à une échelle de proximité, et la mise en œuvre d'une politique publique spécifique avaient suscité une attention particulière sur cette question.

Les projets d'écoquartiers posent donc avec une force toute particulière la question du pilotage et de la gouvernance des opérations urbaines. Nous avons identifié deux types de postures à cet égard chez les maîtres d'ouvrage. L'une s'est manifestée dans un rapport aux habitants principalement envisagé sur un mode hiérarchique et descendant, fondamentalement et exclusivement animé par la préoccupation de maîtrise par ces derniers, de dispositifs éco-techniques permettant une gestion environnementale plus vertueuse. Le principe «d'appropriation du projet » est alors essentiellement assimilé à un principe «d'adhésion» ou «d'acceptabilité sociale». Il donne lieu à des actions d'informations orientées d'une part, vers l'explicitation des « règles de fonctionnement » du quartier déterminées par les « experts patentés », et d'autre part, vers la légitimation des décisions prises par les acteurs politiques et techniques.

Une autre posture est souvent à l'œuvre lorsque les représentants des collectivités associent aux préoccupations environnementales, des enjeux sociaux et la recherche d'un «bien vivre ensemble ». Les élus et techniciens tendent alors à s'engager dans des démarches d'implication des habitants plus importantes. Celles-ci passent généralement le stade consultatif en organisant des dispositifs de concertation et de co-production pour l'aménagement et la gestion d'espaces publics restreints, de parcs urbains ou de jardins partagés. Les niveaux d'implication des habitants les plus élevés observés ont donné lieu 
à des dispositifs portant sur les processus de définition des contenus et sur la formalisation des projets. Ainsi se dévoile une approche de l'appropriation où l'habitant est davantage investi d'un rôle actif, devenant co-décisionnaire de son cadre de vie.

La nécessité de faire évoluer les modalités de décision et d'action pour intégrer une dimension participative dans la poursuite d'objectifs liés au développement durable présente une difficulté considérable pour les collectivités locales. Confrontés à un manque d'expériences déjà constituées sur la question - ou difficilement transférables -, les maîtres d'ouvrage urbains sont contraints d'interroger et adapter leurs pratiques « chemin faisant». Les démarches les plus ambitieuses appréhendent l'opération comme un processus d'expérimentation, dans la perspective d'un apprentissage à la fois pour les élus, les techniciens et les habitants, d'une gouvernance élargie du projet (Fenker, 2015). Mais la conduite de ces expérimentations suppose des conditions qu'ont aujourd'hui du mal à réunir un grand nombre de collectivités françaises, rendant leurs tentatives, ou celles d'habitants porteurs d'initiatives, particulièrement difficiles.

Une première condition renvoie à la culture démocratique de la collectivité, qui s'exprime en particulier à travers des relations qui se sont tissées pendant plusieurs décennies entre les élus et la société civile locale. Les expériences de débat public et les formes de collaboration, voire de porosité, qui se sont développées entre les milieux politique et associatif dans un temps long, transcendant les jeux d'alternance des majorités municipales, sont propices à assigner un objectif politique à la participation, pour considérer les habitants non pas seulement comme des usagers d'un territoire, mais comme des citoyens ayant le droit de s'exprimer, d'être force de propositions concernant un projet qui concerne leur cadre de vie (Blondiaux, 2002; Neveu, 2011). Elles contribuent à dépasser la crainte du conflit qui paralyse encore manifestement beaucoup les plus anciennes générations d'élus et de techniciens. Ce contexte favorise dans certaines communes la création d'un climat de confiance, encourageant aussi bien les mobilisations spontanées d'habitants que leur sollicitation dans le cadre d'une démarche formalisée. Toutefois, on note, y compris dans ces milieux locaux ayant fait l'apprentissage du débat public entre élus et citoyens, que les habitants impliqués dans des instances de coproduction ou de codécision sont très majoritairement issus du milieu associatif. Celui-ci reste en France la forme organisationnelle qui autorise le plus facilement l'accès aux lieux d'échanges dans lesquels le projet se définit. Les habitants eux-mêmes ont tendance à intégrer ces règles d'un jeu finalement très institutionnalisé, et comprennent qu'ils doivent se structurer en collectifs ou en associations pour y jouer un rôle, sans pour autant parvenir à accéder à une véritable légitimité « d'acteur » .

Une deuxième condition fondamentale pour le développement de démarches participatives ambitieuses tient aux modalités de portage politique des projets et à l'autonomie décisionnelle de la collectivité. Bien qu'elles aient acquis des compétences en matière d'aménagement, les collectivités locales françaises restent prises dans une administration territoriale complexe, articulant et imbricant des échelles spatiales et logiques sectorielles, ce qui contraint leurs marges de manœuvre, notamment par rapport aux orientations programmatiques qu'elles peuvent définir dans le cadre d'un projet d'aménagement. Selon la qualité des relations qu'elles auront établies avec leur structure intercommunale de référence et la façon dont elles auront négocié les conditions de leur développement en fonction des spécificités de leur territoire, les termes de la participation qu'elles pourront proposer aux habitants vont être plus ou moins larges. Certaines municipalités n'ont aussi tout simplement pas conscience 
de l'enjeu programmatique, et peuvent se laisser imposer, en l'appréhendant comme une aubaine, une opération d'écoquartier par une instance supra-communale ayant défini les principaux objectifs de l'opération à leur place. Engager un dialogue avec la population peut devenir alors très périlleux, malgré une «bonne volonté politique » initiale, surtout si les habitants ont peu été sollicités jusqu'alors dans des projets d'aménagement urbains.

On a ainsi le sentiment que la façon dont les grands objectifs des opérations d'écoquartiers ont été définis, en termes aussi bien qualitatifs que quantitatifs, constitue souvent une sorte de « boîte noire » pour les habitants. Ces objectifs résultent principalement des documents d'urbanisme et d'orientations politiques fixées par les élus. Ils tendent à s'imposer au début d'une opération comme relevant du « non négociable ». Les habitants ont d'autant moins de «prise » sur ces « règles du jeu » programmatiques, que les Schémas de Cohérence Territoriale, les Plans Locaux d'Urbanisme ou encore les Plans Locaux de l'Habitat, paraissent aujourd'hui très faiblement soumis à concertation citoyenne. Ce problème se trouve amplifié par le fait que les diagnostics urbains initiaux à partir desquels sont censés s'engager des opérations particulières, lorsqu'ils ont été prévus, semblent le plus souvent s'apparenter à des états des lieux conduits par des bureaux d'études techniques, ou relèvent de premiers regards sensibles et prospectifs demandés à des maîtres d'œuvre urbains. Dans les deux cas, ils mettent rarement les habitants à contribution.

Une troisième condition renvoie à la « culture du projet » des élus et de leurs services au sein des collectivités. Prises dans des jeux institutionnels complexes et dans une logique séquentielle soutenue par une forte division technique du travail, disposant pour beaucoup de peu de moyens techniques en interne pour définir et mettre en œuvre leur opération, certaines collectivités se trouvent en effet dans l'obligation de s'en remettre à différents partenaires ou prestataires sans s'apercevoir du rôle qui leur incombe tout au long du projet. Face à la complexité opérationnelle, elles peuvent être amenées à déléguer leur responsabilité de maître d'ouvrage à un aménageur pour monter et conduire leur opération, voire à un opérateur privé qui leur proposera d'intégrer d'autres prestations, de promotion et de construction en particulier, pouvant aboutir à la livraison d'un projet urbain essentiellement immobilier « clefs en main ». Certaines collectivités estiment s'être acquittées de leur mission de maître d'ouvrage après avoir défini - parfois en y associant quelques représentants associatifs -, un cahier des charges permettant de désigner un tel prestataire. Elles comptent ensuite sur le grand professionnalisme de celui-ci pour achever le projet conformément à leurs recommandations initiales, parfois également pour gérer les relations avec les habitants du quartier au cours de cette période. Or, les transformations dans les modes d'habiter que les projets d'écoquartiers supposent, nécessitent un soutien permanent du projet par la collectivité, et précisément par les élus : endosser des orientations stratégiques vis-à-vis de partenaires publics ou privés, procéder à des arbitrages intermédiaires, maintenir dans le temps long un dialogue avec la population, travailler aux conditions d'implication de celle-ci dans la vie future du quartier.

On peut donc constater que les politiques nationales pour promouvoir la réalisation d'écoquartiers n'ont pas abouti à une remise en question fondamentale des ingénieries de projet. Elles ont cependant conduit une majorité de collectivités à accorder une place plus importante à la société civile dans la poursuite d'objectifs de durabilité à l'occasion de la réalisation de ces nouveaux objets urbains. La mise en place et le suivi dans le temps de démarches expérimentales pour favoriser le renouvellement des pratiques des 
élus et des services techniques au sein des collectivités n'ont pu s'opérer que si celles-ci sont parvenues à réunir un certain nombre de conditions. Face à l'inégalité des situations des territoires, des orientations politiques et des compétences professionnelles dont elles disposent, les collectivités livrent une image contrastée de leur capacité d'appropriation au sens d'un rôle actif et de co-production - des politiques nationales. L'innovation dans la manière d'organiser l'action urbaine relève alors moins de la taille de la commune, même si de toute évidence un minimum en moyens humains et financiers est nécessaire, que du portage politique et des différentes formes d'expériences acquises dans la durée dont peuvent bénéficier les projets.

\section{L'élaboration d'une Méthode nationale d'Évaluation}

La délicate question de la diffusion des politiques publiques définies à l'échelle nationale, et en particulier au sein des administrations centrales en France, vers les collectivités, dans le cadre d'une décentralisation toujours en construction, s'est posée avec une grande acuité à partir de 2013. Elle s'est plus concrètement cristallisée autour d'une réflexion portant sur la mise en place d'un Observatoire et d'une Méthode nationale d'Évaluation des ÉcoQuartiers. Pour le Comité scientifique, qui est sollicité à ce propos, l'évaluation trouvera son sens et sera éprouvée dans le cadre de l'obtention du label.

Le Ministère de l'Écologie et du Développement Durable ne peut une nouvelle fois s'engager à financer directement l'évaluation des projets d'ÉcoQuartier. À ce titre, et aussi au nom de la décentralisation, il revient aux collectivités elles-mêmes de procéder à ces démarches. Les membres du Comité scientifique soutiennent pleinement à cette perspective. Ils considèrent d'une manière plus générale, qu'évaluation et développement durable sont intrinsèquement liés (Vivien et al., 2013). Ils voient dans l'auto-évaluation un moyen pour les collectivités de suivre l'avancement de leur projet, de vérifier la pertinence de leurs solutions, d'apprécier les conséquences de leurs décisions, de réorienter certaines actions, d'apprécier les performances effectivement réalisées. Autrement dit, ils envisagent une approche de l'évaluation, endo-formative, in itinere, avant tout utile pour les collectivités elles-mêmes.

La direction de l'AD4 relaye à ce sujet une vision différente du cabinet du Ministre de l'Écologie. Celui-ci souhaite privilégier une méthode d'évaluation « ex-post » permettant de mesurer au plus vite et de manière la plus concrète possible, les résultats de la politique publique qu'il a initiée à travers la démarche ÉcoQuartier. La perspective d'accueillir la $21^{\mathrm{e}}$ Conférence sur le Climat à Paris fin 2015 conforte cette volonté. Le ministère entend ainsi faire la démonstration que ces réalisations répondent bien aux engagements internationaux pris par le gouvernement français. Il sélectionne à cet effet des thèmes qu'il considère prioritaires pour élaborer et tester la méthode d'évaluation : ceux liés aux thématiques de la Grille ÉcoQuartier portant sur la gestion de l'eau, de l'énergie et les déchets, c'est-à-dire pour lesquels des résultats mesurables peuvent être présentés et qui permettent de valoriser des éco-techniques environnementales susceptibles d'être exportées. Un groupe de travail d'experts du Centre Scientifique et Technique du Bâtiment est mandaté à cet effet début 2013. Il doit élaborer rapidement des éléments de méthode d'investigation, des indicateurs et des critères qui devront ensuite servir de références pour les autres «engagements » thématiques. 
D’autres groupes de travail, portés par des équipes de recherche liées à certains représentants du Comité scientifique, ou par des experts du Cerema, sont constitués ${ }^{11}$. Ils se réunissent régulièrement pendant deux ans pour échanger sur l'avancée de leurs travaux respectifs, notamment pour évoquer des questions d'ordre méthodologique et épistémologique. Dès le début de ces réunions, notre groupe de travail centré sur le thème du Pilotage et de la Gouvernance des projets soutient qu'il y a peu d'intérêt à envisager une évaluation seulement de type «ex-post » sur ce sujet.

La direction de l'AD4 admet qu'une autre orientation pourra être donnée à l'évaluation pour cette thématique, mais réaffirme pour les autres, la volonté de la direction du ministère de développer une méthode d'évaluation ex-post. Au fil des réunions, les différentes équipes s'interrogent et interpellent les représentants du ministère sur le sens de ce qu'ils sont en train de produire. Ils ont le sentiment que la constitution d'un observatoire et d'une Méthode nationale d'Évaluation des ÉcoQuartiers tend de plus en plus à ne constituer qu'un seul et même projet qui vise à évaluer la performativité d'une politique publique nationale. Le bénéfice que pourraient tirer les collectivités d'un dispositif d'évaluation pour la définition et la mise en œuvre de leurs propres projets, et plus largement pour réorienter leur action à l'échelle locale, n'apparaît pas comme un enjeu majeur du travail en cours pour l'État central.

Tout en continuant de soutenir le principe d'une auto-évaluation par les collectivités, cette position en faveur d'une évaluation ex-post, privilégiant de surcroît des indicateurs de résultats « mesurables », tend à susciter des approches assez normatives dans les méthodes préconisées, comme le constatent elles-mêmes les différentes équipes lors d'une séance de restitution générale en juillet 2015. Il est manifeste qu'une fois de plus, une vision positiviste de l'évaluation risque de s'imposer (Conan, 1998). La question du développement durable pose un certain nombre de dilemmes à cet égard. À l'échelle nationale, l'État doit s'engager vis-à-vis de l'ensemble de la population et des autres pays pour respecter des objectifs sur des émissions de gaz à effets de serre qui s'expriment essentiellement en terme quantitatif. Il doit ensuite faire en sorte que les pouvoirs publics locaux intègrent et déclinent ces ambitions dans leurs projets. Cette façon de procéder est courante dans le management des politiques publiques en France. C'est par la norme, technique et quantitative, que l'État central a pris l'habitude de s'assurer de la «bonne » mise en application de ses objectifs (Foucault, 1997; Gaudin 2004). Mais ces normes ne sont pas seulement « perfomantielles », énonçant des objectifs à atteindre, elles sont souvent aussi procédurales, c'est-à-dire portant sur des «manières de faire » laissant ainsi peu de possibilités d'invention. Les multiples référentiels, recherchant souvent l'exhaustivité des indicateurs et prédéfinissant des critères d'appréciation, que les services de l'État ont produit ou font produire depuis deux siècles, et plus précisément ces dernières années autour des questions environnementales, en donnent une illustration (Charlot-Valdieu, Outrequin, 2012).

De ce fait, l'évaluation est appréhendée en France, au sein des administrations, comme relevant d'un « contrôle de conformité » aux attentes d'une autorité supérieure, en l'occurrence, l'État. Quels que soient les domaines de l'action publique où on a cherché à la géné-

11 Autour des thématiques suivantes: "Pilotage, gouvernance participative et montage financier des opérations" (sous la responsabilité de notre équipe), "Diagnostic et programmation urbaine prenant en compte les potentiels et les contraintes du territoire", "Qualités urbaines", "Favoriser le vivre ensemble", "Retombées socio-économiques et externalités, "Mobilités", "Changement climatique et risques, "Biodiversité". 
raliser, une première fois à la fin des années 60 , puis à partir des années 90 , l'évaluation tend souvent à être perçue avec défiance par ceux qui en font l'objet. ${ }^{12}$ Dans le domaine de l'aménagement urbain, elle n'est toujours pas envisagée par les maîtrises d'ouvrage comme étant intrinsèque aux démarches de projet, sauf sur des aspects liés au financement des opérations. Elle est rarement perçue comme un moyen de « rendre compte aux citoyens » ni comme un vecteur d'apprentissage, mais comme pouvant déboucher sur des sanctions, surtout lorsqu'elle est suscitée par la réglementation. Cette attitude vis-à-vis de l'évaluation, qui s'est de nouveau vérifiée lorsque plusieurs équipes ont testé avec les acteurs locaux leurs dispositifs et outils pour mettre au point la Méthode nationale d'Évaluation, pose à sa manière la question des rapports qui pourront se nouer entre les services de l'État et les collectivités dans le cadre de la diffusion de la méthode.

Les collectivités qui expérimentent le plus en termes de gouvernance, en particulier en cherchant à associer dès l'amont des acteurs de la société civile, sont celles qui sont le plus intéressées à la méthode et pratiquent déjà l'auto-évaluation de leurs démarches de projet, pour chacune d'elle et de manière transversale. Les équipes de projet en question sont très sceptiques vis-à-vis d'une évaluation déléguée à des bureaux d'études extérieurs : elles souhaitent rester maîtres de la mise au point des démarches, des indicateurs, des critères pour tirer au mieux parti de l'évaluation et surtout faire en sorte qu'elle soit bien contextualisée. Elles estiment que la transparence de la démarche, son partage entre plusieurs services, son ouverture à la société civile, en garantiront la qualité et l'objectivité. Pour autant, tous les chefs de projet que nous avons interviewés soulignent que l'évaluation n'est pas une activité clairement identifiée dans leurs missions. Ils ont ainsi le sentiment que ce n'est pas une tâche valorisée.

La démarche ÉcoQuartier témoigne de l'attitude ambivalente des collectivités vis-à-vis de l'État dans le domaine de l'aménagement; malgré la décentralisation qu'elles approuvent par les compétences qui leur sont ainsi dévolues, elles restent encore très demandeuses de soutien, d'accompagnement de la part de l'État; pas seulement au plan financier mais aussi en termes de conseils. Pour autant, on voit se développer depuis quelques années, un phénomène de mise en réseau des expériences locales, entre collectivités. Les enjeux du développement durable auxquels elles doivent répondre et qui les invitent à l'innovation (Forest et Hamdouch, ed., 2015) les y amènent. L'État peut être utilisé comme un facilitateur à cet égard - la démarche ÉcoQuartier en est un exemple - mais il ne fait plus forcément office d'interlocuteur exclusif (Massardier, 2003).

\section{Conclusion}

Notre analyse permet de souligner le caractère pluriel de la construction des politiques publiques en matière de développement urbain durable, s'opérant à la fois au niveau des instances nationales, en l'occurrence, autour de la mise en œuvre du label ÉcoQuartier, et au niveau des opérations urbaines, menées par les collectivités locales en associant une multiplicité d'autres acteurs. Cette modalité d'élaboration des politiques semble tenir - au

12 Voir plus précisément à propos de la réception, par les collectivités locales de l'injonction gouvernementale à l'évaluation dans les années 90, le dossier de la revue Pouvoirs Locaux, n³8/III/1998. 
plan national comme au plan local - à la capacité de mettre en place et de maintenir dans la durée des démarches partenariales et participatives.

Si le corpus de 187 écoquartiers que nous avons étudié témoigne bien d'un élargissement des compétences mobilisées au cours des opérations urbaines et d'une implication croissante de la société civile, dans de nombreuses situations, les capacités de pilotage et de gouvernance semblent encore faire défaut à la maîtrise d'ouvrage publique. Certaines collectivités n'hésitent pas à confier l'organisation de leurs opérations à d'autres acteurs. Or, une délégation de la maîtrise d'ouvrage urbaine vers d'autres acteurs, aménageurs privés, promoteurs, qui, de par leur statut ne partagent pas forcément les mêmes intérêts que ceux de l'État et des collectivités locales, comporte des risques. Ceux-ci sont assez explicites dans le cadre de "concessions d'aménagement», procédure par laquelle une collectivité peut attribuer la maîtrise d'ouvrage urbaine opérationnelle à un aménageur. Ces transferts de responsabilités fragilisent fréquemment les ambitions qualitatives, en particulier sociales et environnementales des projets, lorsque les collectivités ne sont plus en mesure de suivre et de faire valoir leurs préoccupations : les logiques de marché prennent alors l'ascendant. Elles s'expriment notamment à travers la commercialisation immobilière dans le domaine de l'urbanisme et peuvent conduire, par exemple, à transiger sur la place conférée à la voiture dans un l'espace public, à modifier la typologie de l'habitat pour réaliser davantage de maisons individuelles plutôt que du petit collectif dans un bourg rural, à différer la construction d'équipements et d'espaces publics, voire à réduire leur importance au détriment de la vie du quartier.

Un glissement implicite des responsabilités peut aussi s'opérer de la maîtrise d'ouvrage urbaine vers la maîtrise d'œuvre urbaine, incarnée par un architecte-urbaniste. Celui-ci peut devenir le grand orchestrateur des débats au sein du système d'acteurs du projet et se trouver en quelque sorte implicitement amené à porter l'intérêt général en lieu et place du politique, position que certains n'hésitent pas à assumer en sur-interprétant l'article $1^{\mathrm{er}} \mathrm{de}$ la Loi de 1977 sur l'Architecture.

La baisse des dotations de l'État et la libéralisation de l'économie accentuent la nécessité pour de nombreuses collectivités locales de s'en remettre à des acteurs privés, de négocier et de co-construire les ambitions et objectifs qu'elles poursuivent à travers leurs projets.

Les résultats de nos enquêtes dévoilent une corrélation significative entre les dimensions suivantes : la capacité d'une collectivité à s'organiser en interne pour porter un projet urbain dans la durée, dans le cadre d'un système de pilotage et de gouvernance participative dont elle a fixé les règles; le développement de démarches d'évaluation intégrées à chaque opération portées par l'équipe de projet et se déployant entre les opérations sous la responsabilité d'un service missionné à cet égard ; la poursuite d'ambitions importantes et transversales en matière de développement durable cohérentes avec les politiques publiques.

On remarque plus encore que les collectivités, dotées de capacités permettant de concilier ces différents objectifs et enjeux, suscitent des innovations qui peuvent aujourd'hui peser directement dans la fabrication des politiques nationales et accroître les ambitions qu'elles poursuivent en matière de développement durable : la mise en place d'une politique incitative en faveur de l'habitat participatif en fournit un exemple tout à fait probant. Ainsi, les politiques publiques au plan national dépendent de plus en plus non seulement d'une conjoncture internationale, et des accords que les État ont ratifiés à cette échelle, 
mais aussi de dynamiques ascendantes portées au niveau local, voire parfois micro-local par la société civile qui s'organise ensuite en réseaux. Les collectivités, quelle que soit leur obédience politique, peuvent se fédérer autour de certaines de ces actions, les intégrer dans leur politique puis s'organiser en lobbies vis-à-vis des administrations centrales pour faire évoluer le cadre législatif, ce qui a été le cas pour l'habitat participatif (Devaux, 2015).

Au cours des cinq premières années de sa mise en œuvre, la démarche ÉcoQuartier s'est trouvée au cœur de dynamiques d'articulation entre des mouvements descendants et ascendants, de coopérations transversales entre organismes institutionnels et entre collectivités, de concertations impliquant une diversité d'acteurs, y compris de la société civile. Elle a aussi bénéficié d'une conjoncture très favorable liée au Grenelle de l'Environnement et à une restructuration institutionnelle, qui s'est traduite par la mise en place d'un Ministère d'État en charge de l'Écologie, de l'Énergie, de l'Habitat, de la Nature, des Territoires. Les jeunes équipes de professionnels aux profils très divers qui en ont été les principaux artisans ont manifestement mis à profit cette interdisciplinarité, leur engagement vis-à-vis de son objet et le peu de moyens dont ils disposaient, pour innover dans la fabrique d'une politique publique. Mais cette dynamique pourrait n'être qu'une parenthèse car d'autres tendances lourdes du fonctionnement des administrations françaises n'ont pas tardé à refaire leur apparition en 2014 : recloisonnements ministériels par secteur, restructuration des services à partir de logiques corporatistes, fabrication d'outils validés entre représentants d'administrations centrales, repolarisation sur des ambitions technico-environnementales mesurables, soutien financier substantiel apporté principalement à des actions portées par de grands groupes industriels du BTP et valorisables à l'exportation, etc., mettant une fois de plus, à l'épreuve voire en péril, la portée effective d'une politique publique, ainsi que «l'esprit d'une démarche » en co-construction, directement inspirée par les valeurs et les cadres méthodologiques du développement durable.

\section{Bibliographie}

Baudot P-Y., 2014. Le temps des instruments. Pour une socio-histoire des instruments d'action publique In Halpern C., Lascoumes P., Le Gales P. (eds.). L'Instrumentation de l'action publique, Controverses, résistance, effets. Les Presses de Sciences Po, Paris

Berke P., 2002. Does Sustainable Development Offer a new Direction for Planning? Challenges for the Twenthyfirst Century. Journal of Planning Litterature 17 (1), 21-36.

Blondiaux L., 2002. Où en est la démocratie participative locale en France? Le risque du vide. Les cahiers du DSU 35, 9-10.

Bourdin A., 2005. Dynamiques de la maîtrise d'ouvrage urbaine et mutations du contexte local In Frébault J. (dir.), La maîtrise d'ouvrage urbaine, réflexions sur l'évolution des méthodes de conduite des projets à partir des travaux du Club ville - aménagement, Le Moniteur, Paris, 33-44.

Charlot-Varldieu C. et Outrequin P., 2012. Concevoir et évaluer un projet d'écoquartier : avec le référentiel INDI. Le Moniteur, Paris

Conan M., 1998. L'évaluation constructive. Théorie, principes et éléments de méthode. Éditions de l'Aube, Paris

Devaux C., 2015. L'habitat participatif. De l'initiative habitante à l'action publique. PUR, Rennes

Emelianoff C., 2007. Le bilan des agendas 21 en Europe. Alternatives économiques HS 29, 131-134.

Epron J-P. (ed.), 1992. La commande en architecture. Institut Français d'architecture, tome III, SCIC, Mardaga, Bruxelles 
Epstein R., 2013. Politiques territoriales : ce que les appels à projets font aux démarches de projet. La revue Tocqueville XXXIV (2), 91-102.

Foucault M., 1997. Il faut défendre la société. Cours au Collège de France 1975-1976. Seuil, Paris

Fenker M., 2015. La fabrication des écoquartiers comme espace d'apprentissage pour la maîtrise d'ouvrage urbaine In Debizet G., Godier P., Architecture et urbanisme durables : modèles et savoirs. Cahiers RAMAU $n^{\circ} 7,184-200$.

Fenker M. et Zetlaoui-Léger J., (dir.), 2015. Méthode Nationale Évaluation ÉcoQuartiers : Engagements 2 : «la formalisation et la mise en æuvre d'un processus de pilotage et d'une gouvernance élargie d'un projet d'ÉcoQuartier » et Engagements 3: "l'intégration de l'approche en coût global lors des choix d'investissement », LET- Umr LAVUE Cnrs 7218 et Ministère de l'Égalité des Territoires et du Logement.

Forest J. et Hamdouch A., 2015. Quand la ville durable fait l'innovation. Presses universitaires et techniques romandes, Lausanne

Gaudin J-P., 2004, L'Action publique. Sociologie et politique. Presses de Sciences Po et Dalloz, Paris.

Grudet I., 2015. Le moment écoquartier en France, expérimentations et labellisation In Debizet G., Godier P., Architecture et urbanisme durables : modèles et savoirs, Cahiers RAMAU 7, 22 - 37.

Halpern C., Lascoumes P. et Le Gales P. (eds), 2014. L'Instrumentation de l'action publique, Controverses, résistance, effets. Les Presses de Sciences Po, Paris

Lascoumes P., 2011. Des acteurs aux prises avec le «Grenelle Environnement». Ni innovation politique, ni simulation démocratique, une approche pragmatique des travaux du Groupe V. Participations (1), 277-310.

Lascoumes P. et Le Gales P. 2004. Gouverner par les instruments. Presses de Sciences Po, Paris

Lefèvre P. et Sabard M., 2009. Les écoquartiers. L'avenir de la ville durable. Apogée, Rennes.

Massardier G., 2003. Politiques et action publiques. Armand Colin, Paris

Pinson G., 2004. Le projet urbain comme instrument d'action publique In Lascoumes P., Le Gales P., Gouverner par les instruments, Presses de Sciences Po, Paris, 199-233.

Neveu C., 2011. Habitants, citoyens : interroger les catégories In Baqué M H., Sintomer Y. (dir.), La démocratie participative. Histoire et généalogie, La Découverte, Paris, 39-50.

Rosanvallon P., 2008. La légitimité démocratique, les théories de l'intérêt général. Seuil, Paris

Souami T., 2011. Écoquartiers et urbanisme durable. La Documentation Française 981, Paris

Vanier M., 2011. Gouvernance et interterritorialité, même combat? In Territoires et projets, les outils de la gouvernance, $32^{\mathrm{e}}$ Rencontre nationale des agences d'urbanisme, Paris, 18-21.

Villalba B. (ed.), 2009. Appropriations du développement durable. Émergences, diffusions, traductions. Presses universitaires du Septentrion, Villeneuve d'Ascq

Vivien F-D., Lepart J. et Marty P. (eds.), 2013. L'évaluation de la durabilité. Éditions Quae, Paris

Zetlaoui-Léger J., 2013. L'implication des habitants dans les projets d'écoquartiers en France. Vers des démarches intégrées In Biau V., Fenker M., Macaire E. (eds.), L'implication des habitants dans la fabrication de la ville : Métiers et pratiques en question, Cahiers Ramau 6, 239-254.

Zetlaoui-Léger J., (dir.), Fenker M., Héland L., Grudet I., Gardesse C., Weber B., 2013. La concertation citoyenne dans les projets d'éco-quartiers en France: évaluation constructive et mise en perspective européenne. Programme Concertation, Décision, Environnement ( $2^{\mathrm{e}}$ phase), MEDDE, 2 volumes, http:// www.concertation-environnement.fr/index.php/cde2-2008-2012

Zetlaoui-Léger J., (dir.), Fenker M., Thonnart A., Léonet J., 2014. Les démarches processus, procédures dans les premiers projets d'écoquartiers en France. Ministère de l'Égalité des Territoires et du Logement. http://www.e-lettre.developpement-durable.gouv.fr/la-lettre-du-club-ecoquartier/annee-2014/041----/ rubrique26005.html 